\title{
Treatment outcomes after splenectomy with gastric devascularization or balloon-occluded retrograde transvenous obliteration for gastric varices: a propensity score-weighted analysis from a single institution
}

\author{
Ko Oshita ${ }^{1}$ Masahiro Ohira ${ }^{1,2}$ (D) Naruhiko Honmyo ${ }^{1} \cdot$ Tsuyoshi Kobayashi $^{1}$. \\ Eisuke Murakami $^{3,4}$ - Hiroshi Aikata ${ }^{3,4}$ - Yasutaka Baba ${ }^{5}$ Reo Kawano ${ }^{6}$. \\ Kazuo Awai ${ }^{5} \cdot$ Kazuaki Chayama $^{3,4} \cdot$ Hideki Ohdan $^{1}$
}

Received: 4 August 2019/Accepted: 2 June 2020/Published online: 12 June 2020

(C) Japanese Society of Gastroenterology 2020

\begin{abstract}
Background Although balloon-occluded retrograde transvenous obliteration (BRTO) is often selected to treat gastric varices caused by portal hypertension, data comparing BRTO and splenectomy with gastric devascularization $(\mathrm{Sp}+\mathrm{Dev})$ are limited.

Methods From January 2009 to February 2018, 100 patients with gastric varices caused by portal hypertension who underwent Sp $+\operatorname{Dev}(n=45)$ or BRTO $(n=55)$ were included. Overall survival (OS) and the rebleeding rate were calculated using the inverse probability of a treatment weighting-adjusted log-rank test. Independent
\end{abstract}

Electronic supplementary material The online version of this article (https://doi.org/10.1007/s00535-020-01693-9) contains supplementary material, which is available to authorized users.

Masahiro Ohira

mohira@hiroshima-u.ac.jp

1 Department of Gastroenterological and Transplant Surgery, Graduate School of Biomedical and Health Sciences, Hiroshima University, 1-2-3 Kasumi, Minami-ku, Hiroshima 734-8551, Japan

2 Medical Center for Translational and Clinical Research, Hiroshima University Hospital, Hiroshima, Japan

3 Department of Gastroenterology and Metabolisms, Graduate School of Biomedical and Health Sciences, Hiroshima University, Hiroshima, Japan

4 Research Center for Hepatology and Gastroenterology, Hiroshima University, Hiroshima, Japan

5 Department of Diagnostic Radiology, Graduate School of Biomedical and Health Sciences, Hiroshima University, Hiroshima, Japan

6 Center for Integrated Medical Research, Hiroshima University Hospital, Hiroshima, Japan risk factors were identified by Cox regression analysis. Changes in liver function and adverse events after the procedures were analyzed.

Results Patients in the Sp + Dev group tended to have lower platelet counts than those in the BRTO group, but liver function did not differ between these groups. The 5-year OS rates for the $\mathrm{Sp}+\mathrm{Dev}$ and BRTO groups were 73.4 and $50.0 \%(p=0.005)$, respectively. There were no significant differences in rebleeding rates between the two groups. Multivariate analysis showed that serum albumin level $\leq 3.6 \mathrm{~g} / \mathrm{dL}$, prothrombin time\% activity (PT\%) $\leq 80 \%$, and serum creatinine level $\geq 0.84 \mathrm{mg} / \mathrm{dL}$ were poor prognostic factors. Although the $\mathrm{Sp}+\mathrm{Dev}$ group had more short-term complications after procedures, $\mathrm{Sp}+\mathrm{Dev}$ tended to be more effective in improving liver function than BRTO.

Conclusions $\mathrm{Sp}+\mathrm{Dev}$ showed better OS and improvement of liver function compared with BRTO for the treatment of gastric varices caused by portal hypertension.

Keywords Splenectomy · Gastric devascularization · Balloon-occluded transvenous obliteration - Gastric varices · Portal hypertension

\section{Introduction}

Portal hypertension causes gastric variceal bleeding and hypersplenism. Variceal bleeding has high mortality and constitutes a life-threatening complication of portal hypertension [1]. Various methods have been advocated to treat portal hypertension and variceal bleeding such as splenectomy and gastric devascularization (Sp + Dev), transjugular intrahepatic portosystemic shunt, and balloonoccluded retrograde transvenous obliteration (BRTO) 
[2-5]. BRTO is an interventional radiological technique that is commonly used in Japan [6, 7]. BRTO has a higher rate of successful hemostasis and improves hepatic functional reserve [8]. In case of difficulties in intervention treatment, $\mathrm{Sp}+\mathrm{Dev}$ has been shown to provide satisfactory results in some studies [9-11]. However, few studies have compared $\mathrm{Sp}+$ Dev with BRTO.

In this study, the efficacy and safety of $\mathrm{Sp}+\mathrm{Dev}$ were compared to those of BRTO in patients with gastric varices caused by cirrhotic portal hypertension.

\section{Methods}

\section{Patients and study design}

Clinical data were retrospectively collected from 108 patients who had undergone either Sp + Dev or BRTO for gastric varices from January 2009 to February 2018 at a single tertiary hospital in Japan (Hiroshima University Hospital). All patients were diagnosed with gastric varices by endoscopy.

In the BRTO group, patients comprised 20 bleeding cases and 41 prophylactic cases. In the prophylactic cases, the appearance of the red color sign or F3, or rapidly growing varices with a high risk of rupture, was the indication for BRTO [12]. In bleeding cases, those with portosystemic shunts identified by contrast-enhanced computed tomography (CT) after temporary hemostasis with balloon tamponade or endoscopically were indications for BRTO. Endoscopic findings for varices were evaluated according to the general criteria introduced by the Japanese Research Society for Portal Hypertension [13].

In the present study, 47 patients were treated with $\mathrm{Sp}+\mathrm{Dev}$ for gastric varices caused by portal hypertension. The indicators of $\mathrm{Sp}+\mathrm{Dev}$ included difficulty in interventional radiology, thrombocytopenia due to hypersplenism, and severe portal hypertension. In addition, Child-Pugh class $\mathrm{C}$ and refractory ascites were judged not to be operable and were an indication for BRTO. Patients who underwent $\mathrm{Sp}+\mathrm{Dev}$ or BRTO for gastric varices before the study period or those with insufficient data were excluded from both groups. Ultimately, 8 patients were excluded, leaving 100 patients for statistical analysis (Fig. 1). This study adhered to the ethical principles of the Declaration of Helsinki.

\section{Surgical procedure}

The procedures for $\mathrm{Sp}+$ Dev have been described previously [11]. In brief, an antithrombotic catheter was inserted via the jejunal vein to monitor the portal vein pressure immediately after laparotomy by a midline incision or " $\mathrm{L}$ " incision. A transducer was used to measure the portal vein pressure during surgery, and the catheter was removed before the abdominal operative wound was closed. Splenectomy was performed, and the ligation and division of the vessels at the splenic hilum and the spleen were removed through the incision. After splenectomy, the gastrohepatic ligament was opened and devascularization of the lesser curvature of the stomach was performed. The portal vein pressure was measured to determine the extent of devascularization to ensure the portal vein pressure did not increase by $>50 \%$ from the time of laparotomy. CT was performed preoperatively and at 1-week and 6-month follow-ups after surgery or when indicated clinically. In splenectomy, aspirin was routinely used to prevent portal vein thrombosis. If a portal vein thrombus was detected, thrombolytic therapy including heparin, warfarin, and/or antithrombin III administration was initiated.

\section{BRTO procedure}

The procedures for BRTO were the same as those described previously [12]. In brief, a 5-French catheter with balloon (Seleconballoon catheter; Terumo Clinical Supply, Gifu, Japan) was inserted into the draining vein of the portal systemic shunt via the right femoral or right jugular vein under local anesthesia. During balloon occlusion of the outflow vessels, retrograde venography was performed to determine the hemodynamics of the gastric varices and collateral veins. BRTO was commonly performed using 5\% ethanolamine oleate (Oldamin; Takeda Pharmaceutical, Osaka, Japan) (5\% ethanolamine oleate mixed with iopamidol [EOI]) under balloon occlusion. All patients underwent gastrointestinal endoscopy and intravenous contrast-enhanced CT at approximately 1 week after BRTO.

\section{Study end points}

The primary end point in this study was overall survival (OS) of patients in the Sp + Dev group compared to that of patients in the BRTO group. We also analyzed prognostic factors of OS in the whole study population, $\mathrm{Sp}+\mathrm{Dev}$ group, and BRTO group. The secondary end point was the rebleeding rate of patients. Changes in liver function and platelet counts before and after each procedure were compared and measured before each procedure, and at 6 months and 1 year after each procedure. Furthermore, we compared the rates of short-term complications and length of hospital stay. For the Sp + Dev group, surgery-related complications were also investigated. Ascites was defined as the state in which the administration of a diuretic was newly required after each procedure. 


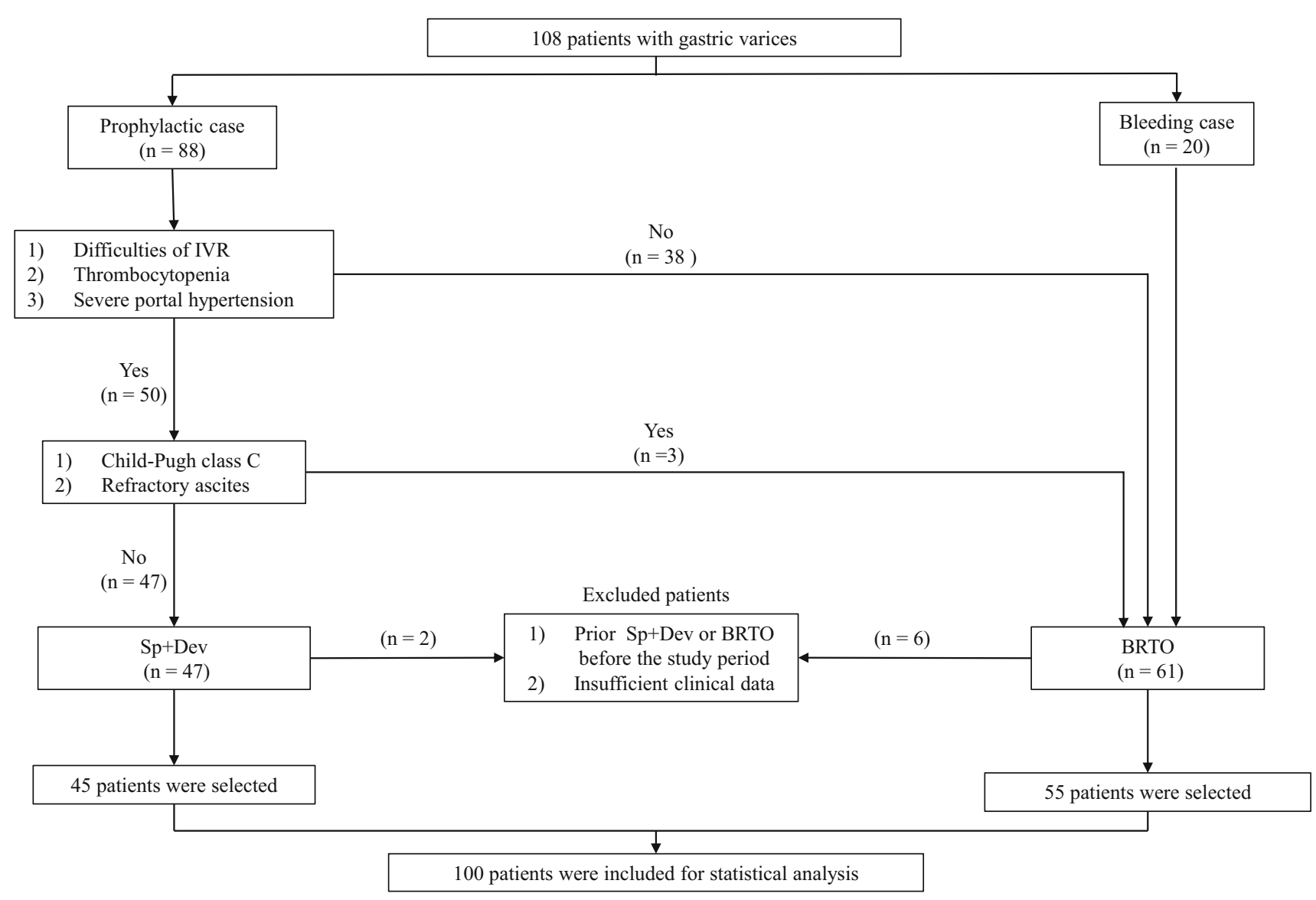

Fig. 1 Flowchart of patient population selection: 108 patients undergoing $\mathrm{Sp}+\mathrm{Dev}$ or BRTO for gastric varices recruited. After excluding 8 patients, 100 patients were included for statistical

This study was conducted in accordance with the 1975 Declaration of Helsinki after receiving approval from the institutional review board of Hiroshima University (Hiroshima, Japan).

\section{Statistical analysis}

To account for differences in baseline characteristics between patients who underwent $\mathrm{Sp}+$ Dev and BRTO, we performed inverse probability of a treatment weighting (IPTW)-adjusted analysis. The probability (or propensity) to undergo $\mathrm{Sp}+\mathrm{Dev}$ versus BRTO was estimated using a logistic regression model based on age, sex, viral hepatitis, concurrent hepatocellular carcinoma (HCC), white blood cell count, hemoglobin level, and serum levels of total bilirubin, albumin, and creatinine. Baseline characteristics were compared between the two groups before and after weighting by using the standardized differences approach, wherein significant imbalances in covariates are present if the standardized difference is $\geq 0.1$ [14]. Propensity score weights were trimmed below and above the 1st and 99th percentiles, respectively [15]. analysis. $S p+D e v$ splenectomy and gastric devascularization, BRTO balloon-occluded retrograde transvenous obliteration, IVR interventional radiology

IPTW-adjusted survivor functions for OS and the rebleeding rate were estimated using the Kaplan-Meier method, and cumulative probabilities of events were compared between the two groups using IPTW-adjusted log-rank tests. A Cox proportional hazards model was used to assess independent risk factors associated with poor OS. Statistically significant variables in the univariate analysis were entered into a multivariate Cox regression model. Changes in liver function and platelet counts were assessed using the paired $t$ test. All statistical analyses were performed using SAS (version 9.4; SAS Institute Inc., Cary, NC, USA). All tests were two sided, and $p<0.05$ was considered statistically significant.

\section{Results}

\section{Patient characteristics and assessment of preoperative liver function}

One hundred patients with gastric varices caused by liver cirrhosis and portal hypertension were divided into two 
groups: $\mathrm{Sp}+\operatorname{Dev}(n=45)$ and BRTO $(n=55)$. Baseline characteristics of the study population are described in Table 1. Before the weighting, the large standardized differences indicated that there was an imbalance between the two groups. Although patients in the $\mathrm{Sp}+\mathrm{Dev}$ were younger and had a better Child-Pugh score, only prothrombin time\% activity (PT\%) was similar in both groups. There were no significant differences between the two groups in terms of the rate of concurrent HCC and staging and treatment method for HCC (Supplementary Table 1). The distributions of most covariates were well balanced between the groups after the IPTW adjustment except for the platelet count and ascites.

Supplementary Fig. 1A shows that the mean portal vein pressure before splenectomy, after splenectomy, and after $\mathrm{Sp}+\mathrm{Dev}$ were $21.6 \pm 4.2 \mathrm{mmHg}, 16.6 \pm 4.2 \mathrm{mmHg}$, and $19.6 \pm 3.9 \mathrm{mmHg}$, respectively. The mean portal vein pressure after $\mathrm{Sp}+$ Dev was significantly lower than that before splenectomy $(p=0.027)$. In contrast, the mean hepatic vein pressure gradient (HVPG) values before and after BRTO were $12.2 \pm 1.0 \mathrm{mmHg}$ and $13.6 \pm 1.0 \mathrm{mmHg}$, respectively (Supplementary Fig. 1B). The mean HVPG after BRTO was higher than that before BRTO, but there was no significant difference $(p=0.150)$.

\section{Overall survival}

The mean follow-up period for the $\mathrm{Sp}+\mathrm{Dev}$ group and BRTO group were 43.4 and 35.4 months, respectively $(p=0.332)$. Figure 2a shows that the OS of the Sp + Dev group was significantly longer than that of the BRTO group $(p=0.005)$. The 1-, 3-, and 5-year OS rates in the Sp + Dev group were $95.2,89.7$, and $73.4 \%$, respectively, while those in the BRTO group were 92.6, 61.5, and 50.0\%, respectively. Next, we used IPTW analysis to compare the OS between the two groups. Figure $2 b$ shows that after IPTW adjustment, patients who underwent $\mathrm{Sp}+\mathrm{Dev}$ had a significantly better OS than those who underwent BRTO $(p=0.026)$.

\section{Predictors of overall survival}

Table 2 shows the results of the univariate and multivariate analyses of prognostic factors of OS in the whole study. In the univariate analysis, age $\geq 66$ years-old, BRTO, white blood cell counts $\leq 3240 / \mathrm{mm}^{\overline{3}}$, hemoglobin level $\leq 10.7 \mathrm{~g} /$ $\mathrm{dL}$, platelet counts $>6.6 \times 10^{4} / \mathrm{mm}^{3}$, serum aspartate transaminase level $\geq 43 \mathrm{IU} / \mathrm{L}$, serum albumin level $\leq 3.6 \mathrm{~g} /$ $\mathrm{dL}, \mathrm{PT} \% \leq 80 \%$, and serum creatinine level $\geq 0.84 \mathrm{mg} / \mathrm{dL}$

Table 1 Baseline characteristics of the patients in the two groups

\begin{tabular}{|c|c|c|c|c|c|c|c|}
\hline \multirow[t]{2}{*}{ Group variables } & \multirow[t]{2}{*}{ All $(n=100)$} & \multicolumn{3}{|c|}{ Before IPTW adjustment } & \multicolumn{3}{|c|}{ After IPTW adjustment } \\
\hline & & $\begin{array}{l}\text { Sp }+ \text { Dev } \\
(n=45)\end{array}$ & $\begin{array}{l}\text { BRTO } \\
(n=55)\end{array}$ & $\begin{array}{l}\text { Std } \\
\text { diff }\end{array}$ & $\begin{array}{l}\text { Sp }+ \text { Dev } \\
(n=45)\end{array}$ & $\begin{array}{l}\text { BRTO } \\
(n=39)\end{array}$ & $\begin{array}{l}\text { Std } \\
\text { diff }\end{array}$ \\
\hline Age (years) & $65.1 \pm 10.0$ & $62.7 \pm 10.7$ & $67.1 \pm 9.0$ & 0.44 & $64.2 \pm 11.0$ & $64.8 \pm 9.1$ & 0.06 \\
\hline Sex (male/female) & $67.0 / 33.0$ & $71.1 / 28.9$ & $63.6 / 26.4$ & 0.16 & $71.7 / 28.3$ & $73.2 / 26.8$ & 0.03 \\
\hline $\begin{array}{l}\text { HBV or HBC/non-B and non- } \\
\text { C }\end{array}$ & $55.0 / 45.0$ & $64.4 / 35.6$ & $47.3 / 52.7$ & 0.35 & $65.0 / 35.0$ & $61.0 / 39.0$ & 0.07 \\
\hline Concurrent HCC & 44.0 & 51.1 & 38.2 & 0.26 & $54.1 / 45.9$ & $50.6 / 49.4$ & 0.07 \\
\hline $\begin{array}{l}\text { White blood cell counts (/ } \\
\mathrm{mm}^{3} \text { ) }\end{array}$ & $5249 \pm 7252$ & $3007 \pm 1043$ & $7084 \pm 943$ & 0.61 & $3647.4 \pm 1566$ & $3686 \pm 1295$ & 0.01 \\
\hline Hemoglobin (g/dL) & $11.0 \pm 2.4$ & $11.8 \pm 0.34$ & $10.4 \pm 0.3$ & 0.61 & $11.7 \pm 1.8$ & $11.6 \pm 1.9$ & 0.07 \\
\hline Platelet counts $\left(\times 10^{4} / \mathrm{mm}^{3}\right)$ & $9.6 \pm 7.8$ & $6.3 \pm 1.1$ & $12.3 \pm 1.0$ & 0.85 & $7.6 \pm 4.4$ & $10.3 \pm 4.7$ & 0.38 \\
\hline Total bilirubin (mg/dL) & $1.4 \pm 0.8$ & $1.2 \pm 0.1$ & $1.5 \pm 0.1$ & 0.28 & $1.2 \pm 0.6$ & $1.3 \pm 0.7$ & 0.09 \\
\hline Albumin (g/dL) & $3.4 \pm 0.7$ & $3.6 \pm 0.1$ & $3.3 \pm 0.1$ & 0.40 & $3.6 \pm 0.4$ & $3.6 \pm 0.6$ & 0.03 \\
\hline $\begin{array}{l}\text { Prothrombin time\% activity } \\
\quad(\%)\end{array}$ & $67.8 \pm 15.6$ & $68.5 \pm 2.3$ & $67.2 \pm 2.1$ & 0.08 & $74.8 \pm 18.2$ & $74.8 \pm 14.9$ & 0.00 \\
\hline Creatinine (mg/dL) & $0.8 \pm 0.2$ & $0.7 \pm 0.0$ & $0.8 \pm 0.0$ & 0.29 & $0.8 \pm 0.2$ & $0.8 \pm 0.2$ & 0.06 \\
\hline Ascites & 7.0 & 8.9 & 5.5 & 0.13 & 6.3 & 1.3 & 0.19 \\
\hline Portosystemic encephalopathy & 9.0 & 6.7 & 10.9 & 0.15 & 4.4 & 6.1 & 0.06 \\
\hline Child-Pugh score & $7.0 \pm 1.6$ & $6.6 \pm 0.2$ & $7.2 \pm 0.2$ & 0.39 & $6.4 \pm 1.0$ & $6.5 \pm 1.6$ & 0.06 \\
\hline
\end{tabular}

Variables are expressed as mean \pm standard deviation or $\%$

$I P T W$ inverse probability of a treatment weighting, Std diff. standardized difference, $S p+D e v$ splenectomy and gastric devascularization, BRTO balloon-occluded retrograde transvenous obliteration, $H B V$ hepatitis B virus, $H C V$ hepatitis $\mathrm{C}$ virus, $H C C$ hepatocellular carcinoma 
(A)

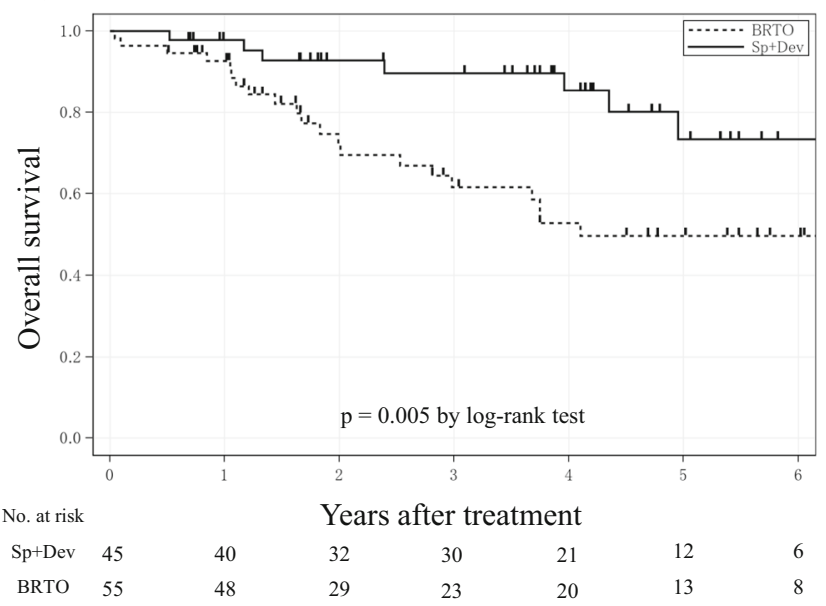

Fig. 2 a Overall survival (OS) in the $\mathrm{Sp}+\mathrm{Dev}$ and BRTO group in all patients. Note the significantly lower OS following $\mathrm{Sp}+\mathrm{Dev}$ compared to BRTO $(p=0.005)$. b OS in the Sp + Dev and BRTO groups after IPTW adjustment. Note a significantly lower OS after

were each significantly predictive of poor prognosis. In the multivariate analysis, serum albumin level $\leq 3.6 \mathrm{~g} / \mathrm{dL}$ (hazard ratio $[\mathrm{HR}]=4.099,95 \%$ confidence interval $[\mathrm{CI}]$ $1.049-19.036 ; p=0.042), \mathrm{PT} \% \leq 80 \%$ (HR $=4.324,95 \%$ CI 1.070-29.699; $p=0.039)$, and serum creatinine level $\geq 0.84 \mathrm{mg} / \mathrm{dL} \quad(\mathrm{HR}=3.229, \quad 95 \% \quad$ CI $\quad 1.524-6.967$; $p=0.002$ ) emerged as independent predictors of poor survival. Furthermore, we assigned a value to these three independent factors of poor survival, which was added to obtain a total risk score ranging from zero to three. Supplementary Fig. 2 shows that the OS did not differ significantly in patients with a risk factor of zero to one between the two groups $(p=0.164)$; however, the OS of patients with a risk factor of two or more in the $\mathrm{Sp}+\mathrm{Dev}$ group was significantly longer than that of patients in the BRTO group $(p=0.004)$. Regarding the cause of death, there were no significant differences except for HCC between the two groups. The $\mathrm{Sp}+\mathrm{Dev}$ group had a significantly higher proportion of HCC deaths than the BRTO group $(p=0.045)$ (Supplementary Table 2$)$.

\section{Rebleeding rate}

During the study period, variceal rebleeding ensued in 14 patients (Sp + Dev: $n=4,8.9 \%$; BRTO: $n=10,18.2 \%$; $p=0.183$ ). As shown in Fig. 3a, the cumulative incidence rate of rebleeding in whole patients did not significantly differ between the two groups $(p=0.124)$. Figure $3 \mathrm{~b}$ shows that the cumulative incidence rate of rebleeding after IPTW adjustment also did not significantly differ between the two groups $(p=0.620)$.

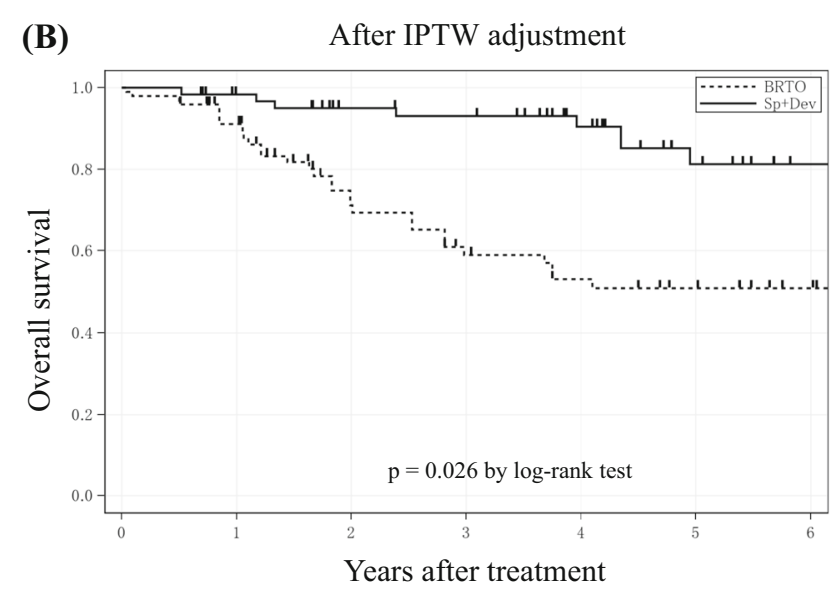

$\mathrm{Sp}+$ Dev compared to the BRTO groups $(p=0.048) . \mathrm{S} p+$ Dev splenectomy and gastric devascularization, BRTO balloon-occluded retrograde transvenous obliteration, IPTW inverse probability of a treatment weighting

\section{Changes in liver function and platelet counts}

Changes in liver function before and after each procedure at 6 and 12 months are shown in Fig. 4. PT\% ( $p=0.011$ at 12 months in the $\mathrm{Sp}+$ Dev group, $p=0.009$ at 12 months in the BRTO group) and serum albumin level ( $p=0.048$ at 12 months in the $\mathrm{Sp}+$ Dev group, $p=0.014$ at 12 months in the BRTO group) significantly increased after each procedure in both groups. However, serum total bilirubin level $(p<0.001$ at 12 months), Child-Pugh score ( $p=0.001$ at 12 months), and platelet counts $(p<0.001$ at 12 months) significantly improved after the procedure only in the $\mathrm{Sp}+\mathrm{Dev}$ group. Although there was no significant difference, Child-Pugh score gradually decreased in patients who underwent BRTO ( $p=0.051$ at 12 months), indicating an improvement in liver function.

\section{Short-term outcome}

Endoscopic evaluation of therapeutic effects and shortterm complications after $\mathrm{Sp}+\mathrm{Dev}$ and BRTO are demonstrated in Table 3. There were no significant differences between the two groups in terms of improvement of gastric varices or eradicating red color sign by endoscopy after the procedures $(p=0.188)$. The incidence of portal vein thrombosis $(p=0.038)$ and ascites $(p<0.001)$ was significantly higher in the Sp + Dev group than in the BRTO group. Pancreatic fistula developed in one (2.2\%) patient who required drainage, and postoperative bleeding occurred in one $(2.2 \%)$ patient who needed reoperation in the $\mathrm{Sp}+\mathrm{Dev}$ group. One (1.8\%) patient died of acute respiratory distress syndrome caused by interstitial pneumonia after BRTO. Hospital stay was significantly longer 
Table 2 Prognostic factors for overall survival identified by univariate and multivariate analyses $(n=100)$

\begin{tabular}{|c|c|c|c|c|c|}
\hline \multirow[t]{2}{*}{ Variables } & \multirow[t]{2}{*}{$n(\%)$} & \multirow{2}{*}{$\begin{array}{l}\text { Univariate } \\
p \text { value }\end{array}$} & \multicolumn{3}{|c|}{ Multivariate } \\
\hline & & & HR & $95 \% \mathrm{CI}$ & $p$ value \\
\hline \multicolumn{6}{|l|}{ Age } \\
\hline$<66$ years & $50(50.0)$ & 0.002 & 1 & & 0.090 \\
\hline$\geq 66$ years & $50(50.0)$ & & 2.026 & $0.899-4.939$ & \\
\hline \multicolumn{6}{|l|}{ Sex } \\
\hline Male & $67(67.0)$ & 0.376 & & & \\
\hline Female & $33(33.0)$ & & & & \\
\hline \multicolumn{6}{|l|}{ Procedure } \\
\hline $\mathrm{Sp}+\mathrm{Dev}$ & $45(45.0)$ & 0.005 & 1 & & 0.730 \\
\hline BRTO & $55(55.0)$ & & 1.253 & $0.361-4.798$ & \\
\hline \multicolumn{6}{|c|}{ Serology of viral hepatitis } \\
\hline HBV or HBC & $55(55.0)$ & 0.732 & & & \\
\hline Non-B and non-C & $45(45.0)$ & & & & \\
\hline \multicolumn{6}{|l|}{ Concurrent $\mathrm{HCC}$} \\
\hline Yes & $44(44.0)$ & 0.289 & & & \\
\hline No & $56(56.0)$ & & & & \\
\hline \multicolumn{6}{|c|}{ White blood cell counts } \\
\hline$\geq 3240\left(\mathrm{~mm}^{3}\right)$ & $57(57.0)$ & & 1 & & 0.642 \\
\hline$<3240\left(\mathrm{~mm}^{3}\right)$ & $43(43.0)$ & 0.036 & 1.303 & $0.441-4.249$ & \\
\hline \multicolumn{6}{|l|}{ Hemoglobin } \\
\hline$\geq 10.7$ (g / dL) & $57(57.0)$ & & 1 & & 0.945 \\
\hline$<10.7$ (g / dL) & $43(43.0)$ & 0.002 & 1.038 & $0.342-2.910$ & \\
\hline \multicolumn{6}{|l|}{ Platelet counts } \\
\hline$<6.6\left(\times 10^{4} / \mathrm{mm}^{3}\right)$ & $33(33.0)$ & 0.020 & 1 & & 0.156 \\
\hline$\geq 6.6\left(\times 10^{4} / \mathrm{mm}^{3}\right)$ & $67(67.0)$ & & 2.438 & $0.726-9.737$ & \\
\hline \multicolumn{6}{|l|}{ Total bilirubin } \\
\hline$<0.8(\mathrm{mg} / \mathrm{dL})$ & $79(79.0)$ & 0.100 & & & \\
\hline$\geq 0.8(\mathrm{mg} / \mathrm{dL})$ & $21(21.0)$ & & & & \\
\hline \multicolumn{6}{|c|}{ Aspartate aminotransferase } \\
\hline$<43(\mathrm{IU} / \mathrm{L})$ & $65(65.0)$ & 0.005 & 1 & & 0.983 \\
\hline$\geq 43$ (IU/L) & $35(35.0)$ & & 1.010 & $0.417-2.544$ & \\
\hline \multicolumn{6}{|c|}{ Alanine aminotransferase } \\
\hline$<29(\mathrm{IU} / \mathrm{L})$ & $57(57.0)$ & 0.091 & 1 & & 0.101 \\
\hline$\geq 29$ (IU/L) & $43(43.0)$ & & 2.382 & $0.844-6.725$ & \\
\hline \multicolumn{6}{|l|}{ Albumin } \\
\hline$>3.6(\mathrm{~g} / \mathrm{dL})$ & $37(37.0)$ & 0.001 & 1 & & 0.042 \\
\hline$\leq 3.6(\mathrm{~g} / \mathrm{dL})$ & $63(63.0)$ & & 4.099 & 1.049-19.036 & \\
\hline \multicolumn{6}{|c|}{ Prothrombin time $\%$ activity } \\
\hline$>80(\%)$ & $19(19.0)$ & 0.045 & 1 & & 0.039 \\
\hline$\leq 80(\%)$ & $81(81.0)$ & & 4.324 & $1.070-29.699$ & \\
\hline \multicolumn{6}{|l|}{ Creatinine } \\
\hline$<0.84(\mathrm{mg} / \mathrm{dL})$ & $64(64.0)$ & 0.013 & 1 & & \\
\hline$\geq 0.84(\mathrm{mg} / \mathrm{dL})$ & $36(36.0)$ & & 3.229 & $1.524-6.967$ & 0.002 \\
\hline
\end{tabular}

$H R$ hazard ratio, $C I$ confidential interval, $H C C$ hepatocellular carcinoma, $S p+D e v$ splenectomy and gastric devascularization, $B R T O$ balloon-occluded transvenous retrograde obliteration, $H B V$ hepatitis $\mathrm{B}$ virus, $H C V$ hepatitis $\mathrm{C}$ virus 
(A) Before IPTW adjustment

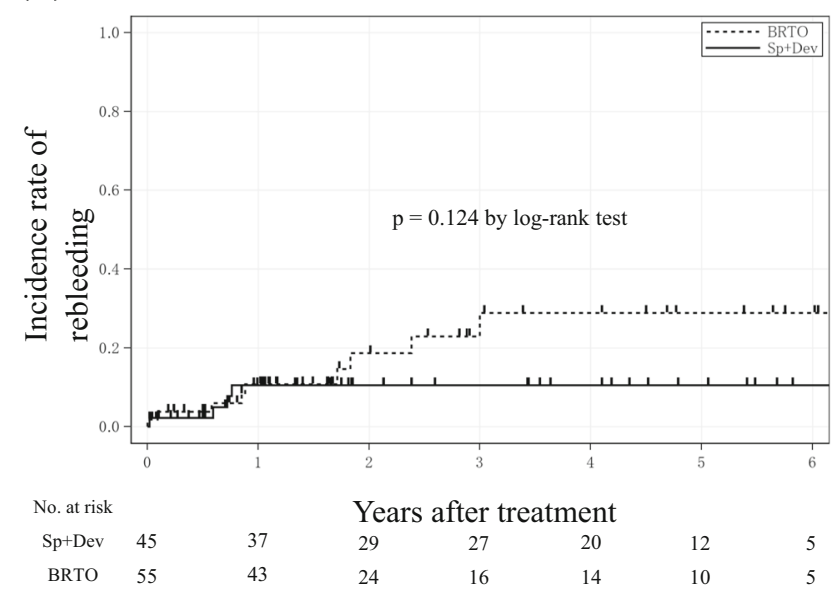

Fig. 3 a The incidence rate of rebleeding in the Sp + Dev and BRTO groups. No significant difference was found between the two groups $(p=0.124)$. b The incidence rate of rebleeding in the $\mathrm{Sp}+\mathrm{Dev}$ and BRTO groups after IPTW adjustment. No significant difference was

in the $\mathrm{Sp}+\mathrm{Dev}$ group than in the BRTO group (17.8 versus 10.5 days, respectively; $p=0.003$ ).

\section{Discussion}

In the present study, we retrospectively investigated the outcome of patients with gastric varices caused by liver cirrhosis and portal hypertension to determine the impact of treatment on clinical outcomes. We found that $\mathrm{Sp}+$ Dev significantly prolonged OS compared with BRTO for all patients and for those selected by IPTW method. In the multivariate analysis, low serum albumin level, low PT\%, and high serum creatinine level were independent prognostic factors of OS. The rate of incidence of rebleeding did not differ between the two groups. Although liver function improved in both groups, Sp + Dev tended to be more effective in improving liver function than BRTO, and thrombocytopenia was improved in only the $\mathrm{Sp}+\mathrm{Dev}$ group. Conversely, $\mathrm{Sp}+\mathrm{Dev}$ demonstrated several disadvantages over BRTO, including a higher incidence of short-term complications and a longer postoperative length of hospital stay.

Although several investigators have reported that survival rates after $\mathrm{Sp}+\mathrm{Dev}$ and BRTO were both favorable, few studies have compared survival outcome at a single institution. The survival rates after $\mathrm{Sp}+\mathrm{Dev}$ reported at 1 , 3 and 5 years ranged from 92.0 to $97.6 \%, 82.0$ to $88.1 \%$, and 64.0 to $76.2 \%$, respectively $[9,16]$. In contrast, the survival rates reported after BRTO at 1,3 and 5 years ranged from 84.7 to $93.0 \%, 76.0$ to $96.5 \%$ and 54.0 to $81.7 \%$, respectively [17-19]. In the present study, the OS for all patients was significantly longer in the $\mathrm{Sp}+\mathrm{Dev}$
(B) After IPTW adjustment

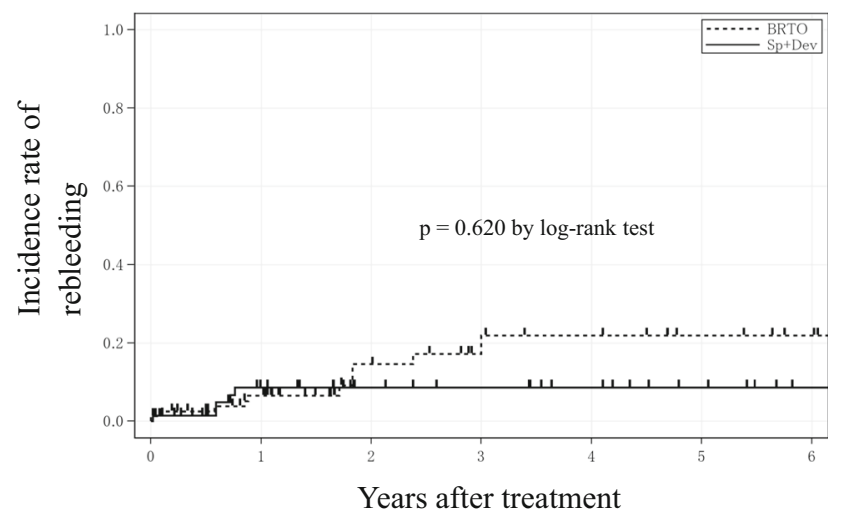

found between the two groups $(p=0.620) . S p+D e v$ splenectomy and gastric devascularization, BRTO balloon-occluded retrograde transvenous obliteration, IPTW inverse probability of a treatment weighting

group than in BRTO the group; however, this result could have been affected by a selection bias of patient. Therefore, we opted to perform IPTW analysis using the propensity score to minimize the impact of selection bias and potential confounding effects. OS, analyzed after IPTW adjustment, was also significantly longer in the $\mathrm{Sp}+\mathrm{Dev}$ group than in the BRTO group. Based on the above results, the $\mathrm{Sp}+$ Dev procedure would be superior to BRTO under similar conditions.

It is well known that the Child-Pugh classification and model for end-stage liver disease score are commonly used as predictors of survival in patients with liver cirrhosis $[20,21]$. The present study showed that low PT\%, low serum albumin level and high serum creatinine level were independent prognostic factors of OS. Gastric varices were also a result of the pathophysiology of liver cirrhosis and portal hypertension; therefore, our results appear to be consistent with previous reports.

Patients with major shunts such as gastric varices have decreased portal blood flow, resulting in a gradually decreased hepatic functional reservoir. A decreased portal blood flow can be recovered by the occlusion of shunts in BRTO, resulting in improvement of liver function [18, 22]. Although the mechanism of improvement of liver function following $\mathrm{Sp}+\mathrm{Dev}$ interventions has yet to be fully elucidated, several studies have reported that splenectomy ameliorates liver function [23, 24]. With regard to hemodynamics in liver cirrhosis, excessive splenic artery blood flowing into spleen causes portal hypertension and a splenohepatic arterial steal syndrome, which leads to the development of a decrease in the hepatic artery blood flow [25]. The portal blood flow decreases after ligation of the splenic artery, thereby decompressing the portal vein 


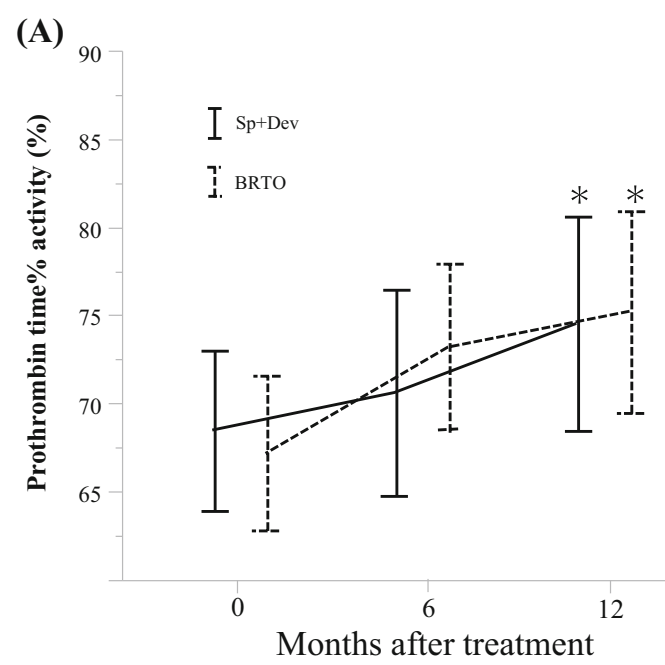

(C)

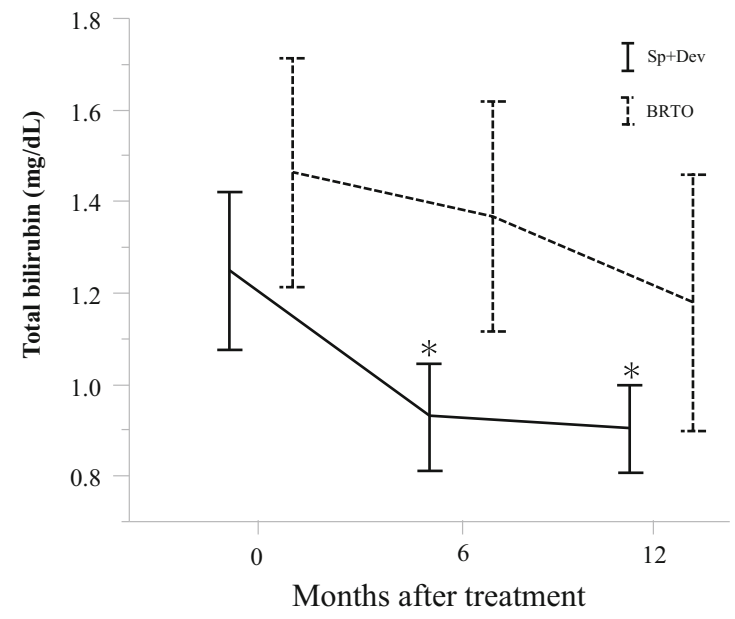

(B)

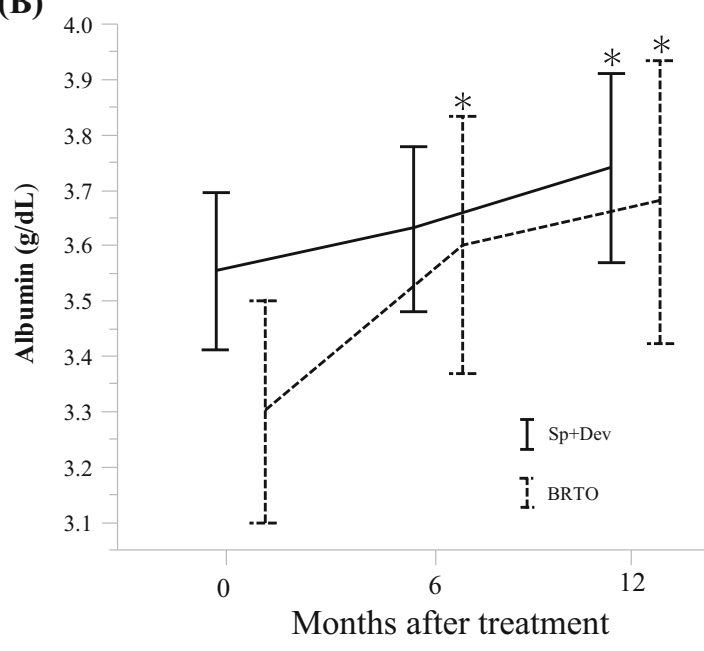

(D)

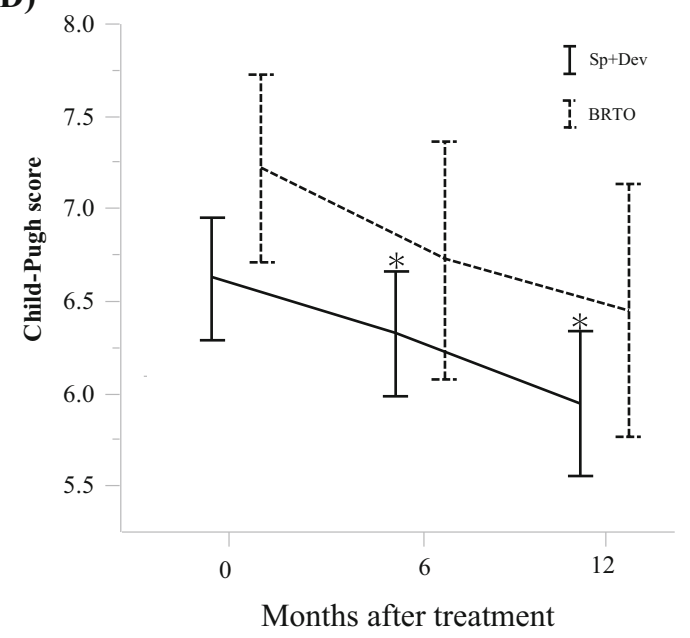

(E)

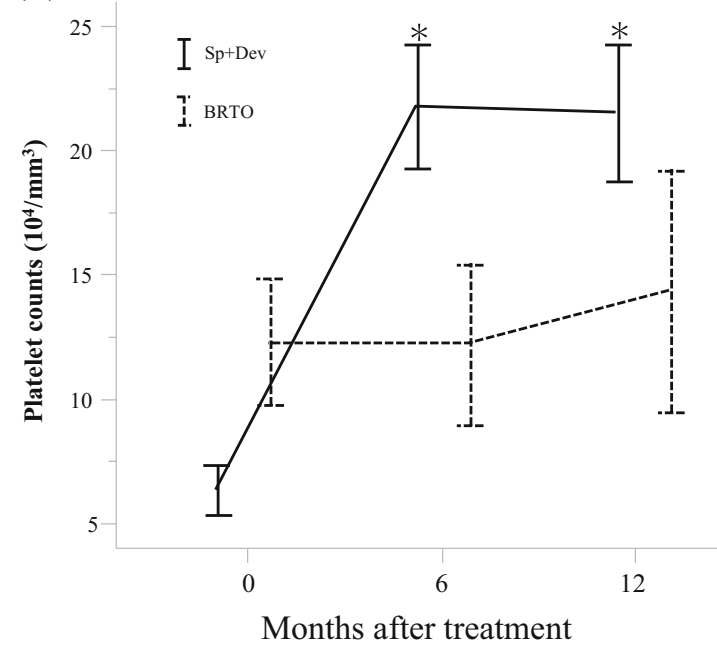


4Fig. 4 Dynamics of liver function after $\mathrm{Sp}+\operatorname{Dev}(n=45)$ and BRTO $(n=55)$ assessed by paired $t$ test: changes in a PT\%, b albumin, c total bilirubin, $\mathbf{d}$ Child-Pugh score, and e platelet counts differed by procedure over time. Variables expressed as the mean \pm 95\% confidence interval. $S p+$ Dev splenectomy and gastric devascularization, BRTO balloon-occluded transvenous obliteration, $P T \%$ prothrombin time $\%$ activity. ${ }^{*} p<0.050$

pressure. Although decreasing the portal vein pressure and increasing hepatic artery blood flow may be associated with improved liver function, no relationship was found between the changes in portal vein pressure by $\mathrm{Sp}+\mathrm{Dev}$ and BRTO and liver function and OS. These results suggest that increasing hepatic blood flow may have a greater effect on improvement of liver function than on reduction of portal vein pressure. Furthermore, various reports have demonstrated the mechanism by which splenectomy improves liver function, including reducing levels of transforming growth factor-beta, which is a hepatocyte growth inhibitor derived from spleen; impairment of overloading the capacity of the liver to metabolize bilirubin; and promoting liver regeneration by preserving the secretion of tumor necrosis factor-alpha [26-28]. Other studies have shown similar evidence in the improvement of liver function after BRTO and splenectomy, but few reports have described the transition of liver function following $\mathrm{Sp}+\mathrm{Dev}$. Although liver function in patients who underwent $\mathrm{Sp}+\mathrm{Dev}$ and BRTO gradually improved over 12 months in the present study, serum total bilirubin level and Child-Pugh score improved significantly only in those who underwent $\mathrm{Sp}+\mathrm{Dev}$. This suggests that $\mathrm{Sp}+\mathrm{Dev}$ is more effective in improving liver function than BRTO.
Furthermore, our study demonstrated that $\mathrm{Sp}+\mathrm{Dev}$ is particularly effective in patients with risk factors.

In addition, the presence of thrombocytopenia has been shown to be a prognostic factor in cirrhotic patients according to its association with significant mortality $[29,30]$. Thrombocytopenia that is associated with liver cirrhosis causes bleeding tendency, difficulties of induction of interferon for hepatitis $\mathrm{C}$ virus (HCV), and difficulties with interventional radiology for HCC. Yamamoto et al. reported that they could perform the treatment of HCC safely for patients who received splenectomy, and this contributed to prolonged OS in patients who underwent splenectomy compared with those who did not undergo splenectomy [31]. Although partial splenic embolization is another effective modality that is less invasive in patients with hypersplenism, some investigators have reported disadvantages such as high recurrence rates of thrombocytopenia and the possibility of infectious complications [32-34]. The Sp + Dev group showed greater improvement of liver function and thrombocytopenia according to splenectomy and increased blood flow into the liver. These factors could be associated with later treatments including direct-acting antivirals for $\mathrm{HCV}$, interventional radiology for HCC and procedures including surgery or biopsy for other diseases due to bleeding tendency. Therefore, patients in the $\mathrm{Sp}+\mathrm{Dev}$ group had a better prognosis than those in the BRTO group.

In the present study, patients in the Sp + Dev group had longer hospital stays and higher rates of complications, including portal vein thrombosis and ascites, than those in the BRTO group. In the treatment of gastric varices, the BRTO group was likely to have included many cases of

Table 3 Post procedure outcome

\begin{tabular}{|c|c|c|c|}
\hline Variables & $\operatorname{Sp}+\operatorname{Dev}(n=45)$ & BRTO $(n=55)$ & $p$ value \\
\hline \multicolumn{4}{|l|}{ Therapeutic effect after procedure $(n, \%)$} \\
\hline Improvement of endoscopic findings for gastric varices & $38(84.4)$ & $51(92.7)$ & 0.188 \\
\hline \multicolumn{4}{|l|}{ Complications $(n, \%)$} \\
\hline Ascites & $22(48.9)$ & $3(5.5)$ & $<0.001$ \\
\hline Portal vein thrombosis & $7(15.6)$ & $2(3.6)$ & 0.038 \\
\hline Splenic vein thrombosis & $1(2.2)$ & $0(0.0)$ & 0.267 \\
\hline Esophageal varices bleeding & $1(2.2)$ & $1(1.8)$ & 0.886 \\
\hline Infection except SSI & $5(11.1)$ & $4(7.3)$ & 0.505 \\
\hline SSI & $9(20.0)$ & - & - \\
\hline Postoperative bleeding & $1(2.2)$ & - & - \\
\hline Pancreatic fistula & $1(2.2)$ & - & - \\
\hline Mortality in hospital stay & $0(0.0)$ & $1(1.8)$ & 0.363 \\
\hline Hospital stay (days) & 17.8 & 10.5 & 0.003 \\
\hline
\end{tabular}

Sp + Dev splenectomy and gastric devascularization, BRTO balloon-occluded transvenous obliteration, SSI surgical site infection 
poor liver function than the $\mathrm{Sp}+\mathrm{Dev}$ group, because the invasiveness of the procedures differs. The cumulative rebleeding rates were similar between the two groups. Essentially, BRTO is often first choice when attempting to eradicate varices because it is less invasive. $\mathrm{Sp}+\mathrm{Dev}$ is performed for patients with BRTO-resistant varices associated with enlarged inflow routes, high-grade portal hypertension, and difficulties in coagulation. However, the surgical procedure has become easy and safe to perform with the recent introduction of a vessel-sealing system and auto-suture devices [23, 35-37]. Together with a trend toward less invasive treatment for portal hypertension, the $\mathrm{Sp}+$ Dev approach for gastric varices with liver cirrhosis is a reasonable treatment option for operable patients.

The limitations of the study include its retrospective nature and that it was performed at a single institution, which may have led to potential bias. However, even after IPTW adjustment, selection bias may not have been avoided completely. Further investigations with a larger number of patients may be necessary.

After IPTW adjustment, we found that $\mathrm{Sp}+\mathrm{Dev}$ significantly prolonged OS compared to BRTO in patients with gastric varices caused by portal hypertension. Besides, the incidence rate of rebleeding did not differ between the $\mathrm{Sp}+$ Dev and BRTO groups, both for the entire study population and after IPTW adjustment. BRTO could reasonably be attempted for gastric varices because it is less invasive than surgery; however, $\mathrm{Sp}+\mathrm{Dev}$ is a useful treatment option that leads to improvements in liver function and thrombocytopenia in operable patients.

Acknowledgements This work was supported in part by the JSPS KAKENHI [Grant Numbers JP17K10669], AMED [Grant Numbers JP18fk0210007, 19fk0310109h0003, and 19fk0210020h0003], and the Takeda Science Foundation. The funders had no role in study design, data collection and analysis, decision to publish, or preparation of the manuscript.

Author contributions KO: contributed to the study design, collected samples, and drafted the manuscript. MO: contributed to the study design, conducted analysis and interpretation of the data, and assisted in drafting and revising the manuscript. NH: collected samples, contributed to the study design, and assisted in drafting and revising the manuscript. TK: contributed to the study design and assisted in drafting and revising the manuscript. EM: collected samples, contributed to the study design, and assisted in drafting and revising the manuscript. HA: contributed to the study design and assisted in drafting and revising the manuscript. YB: contributed to the study design and assisted in drafting and revising the manuscript. RK: conducted analysis of the data and assisted in drafting and revising the manuscript. KA: contributed to the study design and assisted in drafting and revising the manuscript. KC: contributed to the study design and assisted in drafting and revising the manuscript. HO: contributed to the study design and assisted in drafting and revising the manuscript.

\section{Compliance with ethical standards}

Conflict of interest The authors declare that they have no conflicts of interest concerning this article.

\section{References}

1. Grace ND, Groszmann RJ, Garcia-Tsao G, et al. Portal hypertension and variceal bleeding: an AASLD single topic symposium. Hepatology. 1998;28:868-80.

2. Wolff M, Hirner A. Current state of portosystemic shunt surgery. Langenbecks Arch Surg. 2003;388:141-9.

3. Wu X, Ding W, Cao J, et al. Favorable clinical outcome using a covered stent following transjugular intrahepatic portosystemic shunt in patients with portal hypertension. J Hepatobilary Pancreat Sci. 2010;17:701-8.

4. Hashimoto N, Akahoshi T, Yoshida D, et al. The efficacy of balloon-occluded retrograde transvenous obliteration on small intestinal variceal bleeding. Surgery. 2010;148:145-50.

5. Hassab MA. Gastroesophageal decongestion and splenectomy in the treatment of esophageal varices in bilharzial cirrhosis: further studies with a report on 355 operations. Surgery. 1967;61:169-76.

6. Kanagawa H, Mima S, Kouyama H, et al. Treatment of gastric fundal varices by balloon-occluded retrograde transvenous obliteration. J Gastroenterol Hepatol. 1996;11:51-8.

7. Chikamori F, Shibuya S, Takase Y, et al. Transjugular retrograde obliteration for gastric varices. Abdom Imaging. 1996;21:299-303.

8. Hirota S, Kobayashi K, Maeda H, et al. Balloon-occluded retrograde transvenous obliteration for portal hypertension. Radiat Med. 2006;24:315-20.

9. Tomikawa M, Hashizume M, Saku M, et al. Effectiveness of gastric devascularization and splenectomy for patients with gastric varices. J Am Coll Surg. 2000;191:498-503.

10. Liu Y, Li Y, Ma J, et al. A modified Hassab's operation for portal hypertension: experience with 562 cases. J Surg Res. 2013;185:463-8.

11. Tashiro H, Ide $\mathrm{K}$, Amano $\mathrm{H}$, et al. Surgical treatment for portosystemic encephalopathy in patients with liver cirrhosis: occlusion of portosystemic shunt in combination with splenectomy. Hepatol Res. 2013;43:249-54.

12. Naeshiro N, Aikata $\mathrm{H}$, Kakizawa $\mathrm{H}$, et al. Long-term outcome of patients with gastric varices treated by balloon-occluded retrograde transvenous obliteration. J Gastroenterol Hepatol. 2014;29:1035-42.

13. Tajiri T, Yoshida H, Obara K, et al. General rules for recording endoscopic findings of esophagogastric varices (2nd edition). Dig Endosc. 2010;22:1-9.

14. Austin PC. An introduction to propensity score methods for reducing the effects of confounding in observational studies. Multivariate Behav Res. 2011;46:399-424.

15. Austin PC, Stuart EA. Moving towards best practice when using inverse probability of treatment weighting (IPTW) using the propensity score to estimate causal treatment effects in observational studies. Stat Med. 2015;34:3661-799.

16. Kawanaka H, Akahoshi $\mathrm{T}$, Nagao $\mathrm{Y}$, et al. Customization of laparoscopic gastric devascularization and splenectomy for gastric varices based on CT vascular anatomy. Surgical Endosc. 2018;32:114-26. 
17. Ninoi T, Nishida N, Kaminou T, et al. Balloon-occluded retrograde transvenous obliteration of gastric varices with gastrorenal shunt: long-term follow-up in 78 patients. AJR Am J Roentgenol. 2005;184:1340-6.

18. Lee SJ, Kim SU, Kim MD, et al. Comparison of treatment outcomes between balloon-occluded retrograde transvenous obliteration and transjugular intrahepatic portosystemic shunt for gastric variceal bleeding hemostasis. J Gastroenterol Hepatol. 2017;32:1487-94.

19. Akahoshi T, Hashizume M, Tomikawa M, et al. Long-term results of balloon-occluded retrograde transvenous obliteration for gastric variceal bleeding and risky gastric varices: a 10-year experience. J Gastroenterol Hepatol. 2008;23:1702-9.

20. Kamath PS, Wiesner RH, Malinchoc M, et al. A model to predict survival in patients with end-stage liver disease. Hepatology. 2001;33:464-70.

21. D’Amico G, Garcia-Tsao G, Pagliaro L. Natural history and prognostic indicators of survival in cirrhosis: a systematic review of 118 studies. J Hepatol. 2006;44:217-31.

22. Gimm G, Chang Y, Kim HC, et al. Balloon-occluded retrograde transvenous obliteration versus transjugular intrahepatic portosystemic shunt for the management of gastric variceal bleeding. Gut Liver. 2018;11:173-88.

23. Yamamoto J, Nagai M, Smith B, et al. Hand-assisted laparoscopic splenectomy and devascularization of the upper stomach in the management of gastric varices. World $\mathrm{J}$ Surg. 2006;30:1520-5.

24. Ushitora $\mathrm{Y}$, Tashiro $\mathrm{H}$, Takahashi S, et al. Splenectomy in chronic hepatic disorders: portal vein thrombosis and improvement of liver function. Dig Surg. 2011;28:9-14.

25. Quintini C, Hirose K, Hashimoto K, et al. "Splenic artery steal syndrome" is a misnomer: the cause is portal hyperperfusion, not arterial siphon. Liver Transpl. 2008;14:374-9.

26. Murata K, Shiraki K, Sugimoto K, et al. Splenectomy enhances liver regeneration through tumor necrosis factor (TNF)-alpha following dimethylnitrosamine-induced cirrhotic rat model. Hepatogastroenterology. 2001;48:1022-7.

27. Sugawara Y, Yamamoto J, Shimada K, et al. Splenectomy in patients with hepatocellular carcinoma and hypersplenism. J Am Coll Surg. 2000;190:446-50.
28. Nakamura T, Sakata R, Ueno T, et al. Inhibition of transforming growth factor beta prevents progression of liver fibrosis and enhances hepatocyte regeneration in dimethylnitrosamine-treated rats. Hepatology. 2000;32:247-55.

29. Afdhal N, McHutchison J, Brown R, et al. Thrombocytopenia associated with chronic liver disease. J Hepatol. 2008;48:1000-7.

30. Peck-Radosavljevic M. Thrombocytopenia in liver disease. Can J Gastroenterol. 2000;14(Suppl D):60d-6d.

31. Yamamoto N, Okano K, Oshima M, et al. Laparoscopic splenectomy for patients with liver cirrhosis: improvement of liver function in patients with Child-Pugh class B. Surgery. 2015; 158:1538-44.

32. Cai M, Huang W, Lin C, et al. Partial splenic embolization for thrombocytopenia in liver cirrhosis: predictive factors for platelet increment and risk factors for major complications. Eur Radiol. 2016;26:370-80.

33. Zhu K, Meng X, Qian J, et al. Partial splenic embolization for hypersplenism in cirrhosis: a long-term outcome in 62 patients. Dig Liver Dis. 2009;41:411-6.

34. Noguchi H, Hirai K, Aoki Y, et al. Changes in platelet kinetics after a partial splenic arterial embolization in cirrhotic patients with hypersplenism. Hepatology. 1995;22:1682-8.

35. Anegawa G, Kawanaka H, Uehara H, et al. Effect of laparoscopic splenectomy on portal hypertensive gastropathy in cirrhotic patients with portal hypertension. J Gastroenterol Hepatol. 2009;24:1554-8.

36. Kawanaka H, Akahoshi T, Kinjo N, et al. Laparoscopic splenectomy with technical standardization and selection criteria for standard or hand-assisted approach in 390 patients with liver cirrhosis and portal hypertension. $\mathrm{J}$ Am Coll Surg. 2015;221:354-66.

37. Zheng S, Sun P, Liu X, et al. Efficacy and safety of laparoscopic splenectomy and esophagogastric devascularization for portal hypertension: a single-center experience. Medicine. 2018;97:e13703.

Publisher's Note Springer Nature remains neutral with regard to jurisdictional claims in published maps and institutional affiliations. 